\title{
Índice de longitud digital 2D:4D en deportes de pelota 2D:4D Digital Length Index in Ball Sports
}

*Santiago Ramos Bermúdez, *Angélica M. García, *Carlos Federico Ayala, **Haney Aguirre-Loaiza *Universidad de Caldas (Colombia), ${ }^{* *}$ Universidad Católica de Pereira (Colombia)

\begin{abstract}
Resumen. El índice de longitud digital (2D:4D) es un biomarcador del equilibrio entre testosterona T y los estrógenos fetales, por tanto, predictor del desarrollo de masa muscular y hemoglobina, relacionadas con el rendimiento deportivo. Aunque el 2D:4D se ha asociado con el deporte, se requiere comparar el 2D:4D entre deportes. Con el objetivo de establecer diferencias del 2D:4D entre deportistas de deportes de pelota, fueron estudiados 204 deportistas $\left(M_{\text {edad }}=25.9, D E=10.0\right)$, competidores a nivel nacional colombiano e internacional. Se autorreportó talla y masa corporal para calcular Índice de Masa Corporal IMC. También se midió fuerza de prensión manual, longitud de los dedos índice y anular de ambas manos. El estudio fue avalado por comité de bioética. Los resultados del Anova señalaron que el 2D:4D fue similar en ambas. El promedio 2D:4D en ambas manos mostró diferencias $\left[F(9,194)=2.06, p=0.34, c^{2}=.29,1-\hat{a}=.82\right]$. El análisis de longitud por cada dedo mostró diferencias: En la mano derecha $2 \mathrm{Dd}\left[F(9,194)=14.8, p=.00, c^{2}=.63,1-\hat{a}=.95\right], 4 \mathrm{Dd}[F(9$, $\left.194)=12.6, p=.000, c^{2}=.60,1-\hat{a}=.99\right]$. En la mano izquierda, $2 \operatorname{Di}\left[F(9,194)=18.5, p=.000, c^{2}=.68,1-\hat{a}=1.0\right], 4 \operatorname{Di}[F(9,194)=13.2$, $\left.p=.000, c^{2}=.61,1-\hat{a}=.1 .0\right]$. El análisis post-hoc de Tukey-B del 4Dd, 2Di, y 4Di mostró cuatro subconjuntos homogéneos, mientras, el 2Dd cinco subconjuntos. En conclusión, se observaron diferencias en el 2D:4D del promedio de ambas manos. La longitud de los dedos 2Dd-i y 4Dd-i muestra diferencias en los deportes de pelota, principalmente, bádminton, softbol, y squash.
\end{abstract}

Palabras Clave: Talentos; Antropometría; Deportistas; Entrenamiento; Rendimiento, Testosterona.

Abstract. The digital ratio length (2D:4D) is a biomarker of the balance between testosterone (T) and fetal estrogens, therefore, it is a predictor of muscular mass and hemoglobin development, associated with sports performance. Although the 2D:4D has been associated with sports, it is required to compare 2D:4D among sports. With the aim of establishing differences of the 2D:4D between athletes of ball sports, 204 Colombian athletes $\left(M_{a g e}=25.9, S D=10.0\right)$ with national and international competence level were observed. Height and body mass were self-reported in order to calculate Body Mass Index (BMI). Also, strength manual pressure and length of index and ring finger of both hands were measured. This study was approved by the bioethics committee. The Anova results indicated that 2D:4D was similar in both hands. The mean 2D:4D of both hands showed differences $\left[F(9,194)=2.06, p=.034, c^{2}=.29,1-\hat{a}=.82\right]$. The analysis of each finger showed differences: In the right-hand $(\mathrm{r}), 2 \operatorname{Dr}\left[F(9,194)=14.8, p=.000, \varsigma^{2}=.63,1-\hat{a}=.95\right], 4 \operatorname{Dr}[F(9,194)=12.6, p=$ $\left..000, c^{2}=.60,1-\hat{a}=.99\right]$. In the left-hand (1), $2 \mathrm{Dl}\left[F(9,194)=18.5, p=.000, c^{2}=.68,1-\hat{a}=1.0\right], 4 \mathrm{Di}\left[F(9,194)=13.2, p=.000, c^{2}=.61,1-\right.$ $\hat{a}=.1 .0]$. The post-hoc Tukey-B analysis of the $4 \mathrm{Dr}$ and $2 \mathrm{Dr}-4 \mathrm{Dl}$ fingers showed four homogenous subsets, while the 2Dr five subsets. In conclusion, differences of the 2D:4D mean of both hands were observed. The fingers length 2D-4Dr-1 showed differences among ball sports, mostly, badminton, softball, and squash.

Keywords: Talent; Anthropometric; Athletes; Training; Performance; Testosterone.

\section{Introducción}

El índice 2D:4D es un biomarcador del equilibrio entre la Tetosterona (T) y el estrógeno fetal (Manno, 2008). Esta relación queda determinada en una ventana de desarrollo relativamente estrecha al final del primer trimestre del embarazo (Honekopp, Barthold, Beier \& Liebert, 2001; Kim\& Kim, 2016). El 2D:4D es el resultado de dividir la longitud del dedo índice (2D) entre la del dedo anular (4D) de la mano izquierda (i) y derecha (d). Se interpreta que entre menor resulte dicho cociente, mayor influencia androgénica se recibió durante la vida intrauterina. Se dice que la $\mathrm{T}$ fetal y adulta puede ser determinante para el desarrollo de los rasgos físicos, mentales, así como el sistema cardiovascular, tiempo de reacción, agresividad y masculinidad. Estas características atribuidas a la $\mathrm{T}$ se asocian al éxito en las competencias físicas de hombre a hombre, lo que puede determinar la habilidad en los deportes (Tamiya, Lee \& Ohtake, 2016).

Igualmente, la $\mathrm{T}$ se relaciona con mejores desempeños en deportes que requieren mayor esfuerzo físico (Manning $\&$ Hill, 2009). La evidencia disponible sugiere que un bajo 2D:4D se correlaciona con una elevada habilidad atlética

Fecha recepción: 06-03-20. Fecha de aceptación: 26-06-20 Héctor Haney Aguirre Loaiza

haney.aguirre@ucp.edu.co
(Cheng et al., 2015; Hull, Schranz, Manning \& Tomkinson, 2015; Manning, Baron, Wheelwright \& Sanders, 2001; Sudhakar, Majumdar, Umesh, \& Panda, 2014).

Para identificar talentos deportivos y la optimización del rendimiento deportivo desde su complejidad se deben de tener en cuenta diferentes factores: genéticos, físicos, psicológicos, proceso de entrenamiento, posibilidades sociales, y entre otras (Ferriz, Sellés, García \& Cejuela, 2020; Gómez \& Sánchez, 2019),

Se reportó una relación negativa entre el 2D:4D y el rendimiento en carreras de resistencia sobre 110 atletas ( $40 \mathrm{mu}-$ jeres) participantes en competencias (Tamiya, Lee, \& Ohtake, 2012). También se relacionó con el rendimiento en deportes de pelota y con habilidades visuales, espaciales y el desarrollo de un sistema cardiovascular eficiente (Moffit \& Swanik, 2011), además, con capacidades condicionales de velocidad y la resistencia física (Keshavarz, Bayati, Farzad, Dakhili \& Agha-Alinejad, 2017). Otros aportes han sumado evidencia a la relación entre la velocidad y el 2D:4D (Manning \& Hill, 2009); en una muestra de 241 niños mediante la carrera de 50 metros hallaron que quienes tenían menor índice lograban mejores tiempos tanto en los 50 metros como en los parciales de 20, 30 y 40 metros, sugiriendo que el índice 2D:4D es un predictor relativamente débil de las cualidades de fuerza, pero más eficiente en pruebas de velocidad, capacidad determinante en los deportes de pelota. 
Se compararon tenistas colegiales de elite $(27$ hombres, 16 mujeres) con tenistas colegiales no-elite ( 554 hombres, 52 mujeres) y colegiales no-deportistas ( 80 hombres, 86 mujeres), encontrando que, independientemente del sexo, los deportistas tienen un menor 2D:4D que los no-deportistas (Hsu et al., 2015). La relación entre el 2D:4D y el rendimiento competitivo en el baloncesto en 221 hombres australianos de alto rendimiento mostró diferencias significativas para la mano izquierda en función del nivel de competencia (Frick, Hull, Manning, Tomkinson \& Grant, 2017).

De este modo, la evidencia señala que un bajo 2D:4D parece asociarse con el rendimiento deportivo. En consecuencia, estos hallazgos pueden tener implicaciones en los procesos de detección, identificación, formación del talento deportivo y selección de deportistas, además de aspectos relacionados con la madurez potencial de los deportistas y las oportunidades para el desarrollo a largo plazo de sus capacidades y aptitudes (Ferriz et al., 2020).

La detección e identificación de talentos para el deporte de alto rendimiento sigue siendo tema central en la teoría y metodología del entrenamiento y un base fundamental del desarrollo competitivo internacional (De Bosscher, et al., 2007). De una correcta detección depende en buena medida el logro de los altos resultados, pues, las aptitudes deportivas se identifican desde las dimensiones corporales (Holway \& Guerci 2012; Leyva, 2010; Valdés, et al., 2015), el nivel de las capacidades de la condición física (Bulatova \& Platonov, 2017; Volkov \& Filin, 1988) y del aprendizaje motor (Weineck, 2005). Aunque también, más recientemente, se están utilizando análisis genéticos (Kikuchi, Nakazato, Min, Ueda \& Shoji, 2014). Teniendo en cuenta que algunas dimensiones corporales y capacidades de la condición física son estables o fuertes, es decir, poco influenciables por el medio, ellas deben ser evaluadas en función de la edad biológica (Holway $\&$ Guerci, 2012) y buscadas desde un principio en los prospectos deportivos. Una de ellas es el 2D:4D, característica estable a lo largo de toda la vida.

En coherencia con la revisión y las implicaciones del 2D:4D en el deporte, los resultados en su conjunto indican la asociación entre el rendimiento deportivo y 2D:4D; sin embargo, aún falta estudiarse si el 2D:4D es diferente en deportes que comparten características técnicas y físico condicionales similares (e.g., deportes de pelota). Por consiguiente y coherentes con el anterior planteamiento, nuestro objetivo fue el estudio de las diferencias en el índice de longitud digital 2D:4D en deportes de pelota: bádminton, baloncesto, béisbol, fútbol, fustal, softbol, squash, tenis, tenis de mesa y voleibol.

\section{Método}

\section{Tipo de estudio}

Fue realizado un estudio cuantitativo, prospectivo de corte transversal.

\section{Participantes}

A través de una muestral intencional, participaron voluntariamente 204 deportistas hombres $\left(M_{\text {edad }}=25.9, D E=10.0\right.$, Min=12, $M a x=74)$ de diez deportes de pelota: Bádminton ( $n=$ $15)$, Baloncesto $(n=35)$, Béisbol $(n=7)$, Fútbol $(n=17)$, Fustal
( $n=18)$, Softbol $(n=12)$, Squash $(n=5)$, Tenis $(n=13)$, Tenis de mesa $(n=14)$ y Voleibol $(n=68)$. Los criterios de inclusión fueron: (a) competir en algún deporte de pelota, y (b) haber competido a nivel nacional y/o internacional.

\section{Procedimiento y materiales}

La evaluación se efectuó en los Juegos Deportivos Nacionales de Colombia, Campeonatos Nacionales de Ligas, y Juegos Nacionales Universitarios, así como en los sitios de concentración y/o entrenamiento entre en seis ciudades colombianas (Bogotá, Cali, Ibagué, Medellín, Manizales, y Palmira) entre 2015 y 2017. La talla(m) y masa corporal(kg) fueron registrados por autorreporte dada la imposibilidad de trasladar equipos adecuados a las sedes de los eventos. Para calcular el índice de masa corporal $=$ peso $/$ talla $^{2}(\mathrm{Kg} /$ $\left.\mathrm{m}^{2}\right)$.

La fuerza de prensión manual se midió con un dinamómetro digital Takei Ltd., modelo Smedly III con precisión 0.1 kgf, ajustable al tamaño de la mano. El protocolo de evaluación fue: el sujeto con el brazo extendido y paralelo al tronco sujetaba el aparato ejerciendo la fuerza máxima durante 3 a 5 segundos. Tras una recuperación de un minuto, se repitió la maniobra alternando la mano. Se anotó el mejor de los dos intentos de cada mano y se apuntó sí el sujeto era diestro o zurdo (Marrodán et al. 2009).

Se midió la longitud de los dedos índice (2Dd-i) y anular (4Dd-i) directamente de las palmas de las dos manos. Se siguió el protocolo descrito por Manning y Taylor (2001). Se usó un calibrador vernier digital marca Sata ${ }^{\circledR}$ modelo 91511, con precisión de $0.01 \mathrm{~mm}$. El 2D:4D se calculó dividiendo la longitud del dedo índice entre la del dedo anular. Se analizaron separadamente los índices de cada mano, el promedio de las dos manos y la diferencia del índice de la mano derecha menos la izquierda, dado a que la mayoría de los antecedentes presentan estos estadísticos separados, y se ha encontrado una mayor relación entre el índice de la mano derecha y los resultados deportivos (Dapeng at al. 2012; Acar, 2018; Hill et al., 2012; Voracek et al., 2006).

\section{Análisis de datos}

Los datos fueron procesados en una matriz de datos de Excel para Windows Office ${ }^{\circledR}$. Seguidamente, con el programa SPSS v.24, IBM ${ }^{\circledR}$. El análisis exploratorio de los datos permitió identificar tres casos como datos extremos, los cuales fueron tratados con la técnica de winsorizing (McGrath, 2011). Se comprobó la normalidad (Kolmogorov-Smirnov) para las variables dependientes 2D:4Dd $[K-S(204)=0.54$, $p=.200], 2 \mathrm{D}: 4 \mathrm{Di}[K-S(204)=0.31, p=.200] \mathrm{y}$ ambas manos $[K-$ $S(204)=0.52, p=.200]$ y homocedasticidad (Levene). Se calcularon frecuencias, medidas de tendencia central (Media) y de dispersión (Desviación Estándar). El análisis inferencial se estimó con análisis paramétrico de un Anova de un factor para estimar las diferencias del 2D:4D entre los deportes. El análisis de comparación de medias se efectuó a través de las pruebas post-hoc de Bonferroni para el 2D:4D y HSD TukeyB para la longitud de cada dedo de cada mano (2Dd, 4Dd y 2Di 4Di). El tamaño del efecto fue calculado a través del Eta cuadrado $\left(\eta^{2}\right)$ (Fritz et al., 2012), cuya magnitud es interpretada así (Cohen, 1988): <.01 pequeño, .06, moderado, >.14 fuerte. La potencia estadística $(1-\beta)$ fue estimada a través del 
software de G*Power (Faul et al., 2009).

\section{Consideraciones éticas}

Los evaluados firmaron un asentimiento informado y los padres o delegados de equipos el consentimiento informado en el caso de los menores de edad ( $<18$ años). El estudio fue avalado por el Comité de Bioética de la Facultad de Ciencias para la Salud de la Universidad de Caldas (Acta 009/2015), de acuerdo con la Declaración de Helsinki. Se siguieron los lineamientos de la investigación con seres humanos disponiendo de principios de la integridad, autonomía y respeto por los participantes.

\section{Resultados}

Los datos descriptivos de las variables antropométricas y la fuerza de prensión manual se encuentran en la tabla 1.

La tabla 2 muestra los datos descriptivos y el análisis de varianza del 2D:4D según los deportes.

Se observaron diferencias significativas en las medias de ambas manos, $\left[F(9,194)=2.06, p=0.34, \eta^{2}=.29,1-\beta=.82\right]$; sin embargo, el análisis post-hoc de Bonferroni y Tukey-B no estimó diferencias de las medias, ni subconjuntos homogéneos. El 2D:4D de la mano izquierda, 2D:4D de la mano derecha, y diferencia entre ambas manos no mostraron efectos significativos.

De acuerdo con la tabla 3, la longitud por cada dedo mostró diferencias significativas en ambas manos. Los tamaños del efecto se estimaron como fuertes. El análisis posthoc a través de Tukey-B se observa en las tablas 4 y 5 . En la mano derecha, la longitud del 2Dd mostró cinco subconjuntos homogéneos, y el 4Dd cuatro subconjuntos. Respecto a la mano izquierda, ambos dedos (2Di y 4Di) mostraron cuatro subconjuntos homogéneos.

\section{Discusión}

El objetivo fue establecer las diferencias del índice 2D:4D en deportistas de diferentes deportes de pelota, participantes en eventos deportivos colombianos realizados en los años 2015 y 2016. Los datos muestran tres resultados principales: Primero, el 2D:4D de las manos derecha e izquierda fue homogéneo. Segundo, el 2D:4D promedio de ambas manos mostró diferencias significativas en los deportes, sin embargo, el análisis post-hoc no discrimina las diferencias entre las medias de las disciplinas deportivas. Por consiguiente, este hallazgo debe ser asumido con cautela. Y tercero, el análisis de longitud de los dedos mostró diferencias significativas en los deportes de pelota. De tal modo que el análisis post-hoc de los dedos 4Dd, 2Di, y 4Di mostraron cuatro subconjuntos homogéneos, en tanto, el 2Dd cinco subconjuntos.

En coherencia con los anteriores resultados, también es importante resaltar el valor descriptivo observado en el 2D:4D frente a los deportes de pelota. El análisis de la mano derecha mostró que el mejor promedio fue para los deportistas de bádminton, seguidos en orden a squash, softbol, futsal y voleibol. En cuanto a la mano izquierda el orden fue: bádminton, tenis de mesa, softbol, squash, tenis y voleibol. En el promedio de ambas manos el orden fue bádminton,
Tabla 1.

Descriptivos $(\mathrm{M} / \pm)$ de las variables antropométricas, dinamometría segín lus discipling deportivas. \begin{tabular}{llllll}
\hline Deporte & $n$ & Edad & Talla & Masa Corporal IMC & Dinamometría
\end{tabular} \begin{tabular}{lcccccc} 
& & $($ años$)$ & $(\mathrm{cm})$ & $(\mathrm{kg})$ & $\left(\mathrm{kg} / \mathrm{m}^{2}\right)$ & $(\mathrm{Kgf})$ \\
\hline 1.Badminton $^{(\mathrm{a})}$ & 15 & $18.6 \pm 3.8$ & $164.6 \pm 10.7$ & $57.3 \pm 12.3$ & $20.8 \pm 2.3$ & $37.3 \pm 10.2$
\end{tabular} $\begin{array}{lcccccc}\text { 2.Baloncesto } & 35 & 34.6 \pm 15.4 & 181.3 \pm 8.4 & 81.9 \pm 11.6 & 24.8 \pm 3.2 & 43.2 \pm 9.1\end{array}$

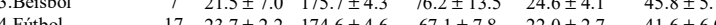
$\begin{array}{lllllll}\text { 4.Futbol } & 17 & 23.7 \pm 2.2 & 174.6 \pm 4.6 & 67.1 \pm 7.8 & 22.0 \pm 2.7 & 41.6 \pm 6.0\end{array}$ $\begin{array}{llllll}\text { 5.Futsala } & 18 & 25.4 \pm 2.0 & 173.2 \pm 7.1 & 66.8 \pm 7.6 & 22.3 \pm 2.6\end{array}$

$\begin{array}{lcccccc}\text { 6.Softbol } & 12 & 37.5 \pm 14.4 & 172.0 \pm 5.6 & 85.3 \pm 10.7 & 28.8 \pm 3.5 & --\end{array}$

$\begin{array}{lcccccc}\text { 7.Squash } & 5 & 24.4 \pm 2.4 & 172.4 \pm 2.3 & 66.6 \pm 8.3 & 22.3 \pm 2.3 & 51.1 \pm 8.0 \\ \text { 8. Tenis } & 13 & 24.7 \pm 5.6 & 179.4 \pm 8.2 & 74.9 \pm 11.1 & 23.3 \pm 2.1 & 43.0 \pm 8.1\end{array}$ $\begin{array}{lllllll}\text { 8. Tenis } & 13 & 24.7 \pm 5.6 & 179.4 \pm 8.2 & 74.9 \pm 11.1 & 23.3 \pm 2.1 & 43.0 \pm 8.1\end{array}$ $\begin{array}{lllllll}\text { 9. Tenis de mesa }{ }^{(b)} & 14 & 19.5 \pm 5.5 & 167.6 \pm 11.9 & 59.7 \pm 9.1 & 22.1 \pm 1.5 & 35.3 \pm 11.0\end{array}$ \begin{tabular}{lcccccc} 
10. Voleibol & 68 & $23.4 \pm 5.8$ & $189.5 \pm 8.4$ & $79.5 \pm 10.5$ & $21.9 \pm 2.3$ & $48.8 \pm 9.0$ \\
\hline
\end{tabular}

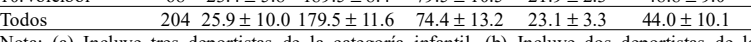
Nota: (a) Incluye tres deportistas de la categoría infantil, (b) Incluye dos deportistas de la categoría infantil. Futsal y Softbol no reportaron datos de dinamometría

Tabla 2.

Promedio (M) y desviación típica $( \pm)$ del índice 2D:4D según deportes.

\begin{tabular}{|c|c|c|c|c|c|}
\hline \multirow{3}{*}{ Deporte } & \multicolumn{5}{|c|}{ 2D:4D } \\
\hline & $n$ & Mano & Mano & Media & Diferencia \\
\hline & & Derecha & Izquierda & Dos manos & \\
\hline 1.Badminton & 15 & $.927 \pm .034$ & $.954 \pm .043$ & $.934 \pm .030$ & $-.046 \pm .082$ \\
\hline 2. Baloncesto & 35 & $.953 \pm .032$ & $.976 \pm .043$ & $.965 \pm .032$ & $-.023 \pm .043$ \\
\hline 3.Beisbol & 7 & $.952 \pm .048$ & $.971 \pm .065$ & $.961 \pm .056$ & $-.019 \pm .020$ \\
\hline 4.Fútbol & 17 & $.959 \pm .037$ & $.976 \pm .043$ & $.967 \pm .036$ & $-.017 \pm .036$ \\
\hline 5.Futsala & 18 & $.950 \pm .044$ & $.983 \pm .033$ & $.966 \pm .035$ & $-.033 \pm .035$ \\
\hline 6.Softbol & 12 & $.935 \pm .028$ & $.948 \pm .034$ & $.942 \pm 026$ & $-.014 \pm .035$ \\
\hline 7.Squash & 5 & $.935 \pm .025$ & $.955 \pm .036$ & $.945 \pm .025$ & $-.021 \pm .036$ \\
\hline 8.Tenis & 13 & $.961 \pm .052$ & $.969 \pm .036$ & $.965 \pm .036$ & $-.008 \pm .051$ \\
\hline 9. Tenis de mesa & 14 & $.952 \pm .032$ & $.947 \pm .039$ & $.950 \pm .024$ & $-.005 \pm .054$ \\
\hline 10.Voleibol & 68 & $.951 \pm .041$ & $.969 \pm .036$ & $.960 \pm .033$ & $-.018 \pm .041$ \\
\hline Total & 204 & $.948 \pm .041$ & $.968 \pm .040$ & $.959 \pm .033$ & $-.020 \pm .046$ \\
\hline Anova & & $F(9,194)=2.04$ & $F(9.194)=1.67$ & $F(9.194)=2.06$ & $F(9.194)=1.33$ \\
\hline$p$ & & $p=.310$ & $p=.096$ & $p=.034$ & $p=.222$ \\
\hline$\eta^{2}$ & & .23 & .26 & .29 & .24 \\
\hline $1-\beta$ & & .48 & .71 & .82 & .64 \\
\hline
\end{tabular}
organización los deportes de menor a mayor

Tabla 3

Análisis de Varianza de la longitud(mm) de cada dedo por cada mano según el deporte

\begin{tabular}{|c|c|c|c|c|c|}
\hline \multirow{2}{*}{ Deporte } & \multirow[b]{2}{*}{$n$} & \multicolumn{2}{|c|}{ Mano Derecha } & \multicolumn{2}{|l|}{ Mano Izquierda } \\
\hline & & 2Dd & 4Dd & $2 \mathrm{Di}$ & 4Di \\
\hline 1.Badminton & 15 & $64.7 \pm 5.5$ & $71.0 \pm 4.1$ & $65.6 \pm 4.3$ & $68.5 \pm 4.2$ \\
\hline 2.Baloncesto & 35 & $75.8 \pm 5.1$ & $79.5 \pm 4.8$ & $77.4 \pm 4.4$ & $79.4 \pm 5.0$ \\
\hline 3.Beisbol & 7 & $74.6 \pm 1.9$ & $77.5 \pm 3.5$ & $75.0 \pm 2.0$ & $76.3 \pm 4.2$ \\
\hline 4.Fútbol & 17 & $70.8 \pm 4.6$ & $74.2 \pm 4.0$ & $71.5 \pm 4.2$ & $73.7 \pm 4.3$ \\
\hline 5.Futsala & 18 & $71.6 \pm 5.6$ & $75.4 \pm 5.8$ & $73.0 \pm 4.5$ & $74.4 \pm 5.0$ \\
\hline 6.Softbol & 12 & $71.8 \pm 3.0$ & $76.8 \pm 3.2$ & $73.0 \pm 3.2$ & $77.0 \pm 3.5$ \\
\hline 7.Squash & 5 & $72.3 \pm 1.1$ & $77.4 \pm 1.8$ & $74.5 \pm 1.8$ & $78.0 \pm 2.6$ \\
\hline 8.Tenis & 13 & $74.7 \pm 3.9$ & $77.9 \pm 3.8$ & $74.7 \pm 3.6$ & $77.2 \pm 4.7$ \\
\hline 9. Tenis de mesa & 14 & $68.1 \pm 5.4$ & $71.5 \pm 5.5$ & $68.7 \pm 5.1$ & $72.6 \pm 6.5$ \\
\hline 10. Voleibol & 68 & $77.8 \pm 5.2$ & $81.8 \pm 5.7$ & $78.7 \pm 5.1$ & $81.3 \pm 5.7$ \\
\hline Total & 204 & $73.9 \pm 6.3$ & $77.9 \pm 6.1$ & $74.9 \pm 6.0$ & $77.4 \pm 6.3$ \\
\hline Anova & & $F(9,194)=14.8$ & $F(9,194)=12.6$ & $F(9,194)=18.5$ & $F(9,194)=13.2$ \\
\hline$p$ & & .000 & .000 & .000 & .000 \\
\hline$\eta^{2}$ & & .63 & .60 & .68 & .61 \\
\hline $1-\beta$ & & 0.95 & 0.99 & 1.0 & 1.0 \\
\hline
\end{tabular}
estadística Promedio $(M)$ y desviación típica $( \pm)$

Tabla 4,

Análisis post-hoc de la longitud ( $\mathrm{mm}$ ) de los dedos de la mano derecha

\begin{tabular}{|c|c|c|c|c|c|c|c|c|c|c|}
\hline \multirow{2}{*}{ Deporte } & \multicolumn{5}{|c|}{ Subconjuntos - 2Dd } & \multirow{2}{*}{ Deporte } & \multicolumn{4}{|c|}{ Subconjuntos - 4Dd } \\
\hline & I & II & III & IV & $\mathrm{V}$ & & I & II & III & IV \\
\hline Badminton & 65.6 & & & & & Badminton & 68.5 & & & \\
\hline Tenis de mesa & 68.7 & 68.7 & & & & Tenis de mesa & 72.6 & 72.6 & & \\
\hline Fútbol & & 71.5 & 71.5 & & & Fútbol & 73.7 & 73.7 & 73.7 & \\
\hline Softbol & & 73.0 & 73.0 & 73.0 & & Futsal & 74.4 & 74.4 & 74.4 & \\
\hline Futsala & & 73.0 & 73.0 & 73.0 & & Beisbol & & 76.3 & 76.3 & 76.3 \\
\hline Squash & & & 74.5 & 74.5 & 74.5 & Softbol & & 77.0 & 77.0 & 77.0 \\
\hline Tenis & & & 74.7 & 74.7 & 74.7 & Tenis & & 77.2 & 77.2 & 77.2 \\
\hline Beisbol & & & 75.0 & 75.0 & 75.0 & Squash & & 78.0 & 78.0 & 78.0 \\
\hline & & & & 77.4 & 77.4 & Baloncesto & & & 79.4 & 79.4 \\
\hline Voleibol & & & & & 78.7 & Voleibol & & & & 81.3 \\
\hline
\end{tabular}

Nota: El valor reportado es la Media de la longitud (mm) del dedo.

Tabla 5 .

Análisis post-hoc de la longitud(mm) de los dedos de la mano izquierda Deporte Subconjuntos - 2Di Deporte

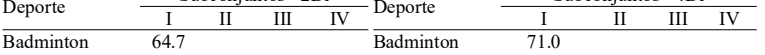
$\begin{array}{lllllll}\text { Tenis de mesa } & 68.1 & 68.1 & & \text { Tenis de mesa } & 71.5 & 71.5\end{array}$ $\begin{array}{lllllll}\text { Fútbol } & 70.8 & 70.8 & \text { Fútbol } & 74.2 & 74.2 & 74.2\end{array}$ $\begin{array}{llllllll}\text { Futsala } & 71.6 & 71.6 & \text { Futsala } & 75.4 & 75.4 & 75.4\end{array}$

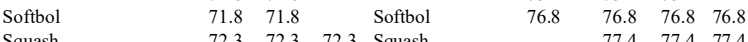
$\begin{array}{llllllll}\text { Squash } & 72.3 & 72.3 & 72.3 & \text { Squash } & 77.4 & 77.4 & 77.4 \\ \text { Beisbol } & & 74.6 & 74.6 & \text { Beisbol } & 77.5 & 77.5 & 77.5\end{array}$ $\begin{array}{llllrrr}\text { Beisbol } & 74.6 & 74.6 & \text { Beisbol } & 77.5 & 77.5 & 77.5 \\ \text { Tenis } & 74.7 & 74.7 & \text { Tenis } & & 77.9 & 77.9 \\ \text { Bas } & 75.8 & 75.8 & \text { Bes } & & 79.5 & 79.5\end{array}$

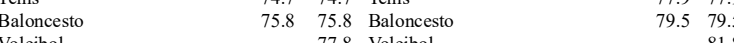
Voleibol Nota: El valor reportado es la Media de la longitud (mm) del dedo.

tenis de mesa, softbol, squash, y voleibol. El análisis de frecuencias indica que los deportistas de bádminton, softbol, y squash siempre estuvieron con el mejor promedio del 2D:4D, indicando buena exposición a la testosterona en la etapa pre-natal. El promedio total de ambas manos fue de $0.959 \pm 0.33$.

Los deportistas de bádminton, softbol, y squash estuvieron presentes entre los mejores promedios del 2D:4D. 
Estos deportes comparten características técnicas, físico condicionales y psicológicas similares. Por ejemplo, las exigencias del control inhibitorio y situaciones de destreza amplia (Gabbett et al., 2008). Diversas situaciones competitivas entre el objeto (pelota, raqueta, etc.), el oponente y el deportista demandan respuestas rápidas y de un alto nivel de precisión. En general, el tiempo de reacción y la precisión son características a la agilidad en el rendimiento deportivo (Paul et al., 2016). Así mismo, en este orden ideas, es importante considerar reportes previos los cuales han advertido sobre las diferencias en las características psicológicas de deportes de pelota y de conjunto (Arias et al., 2016).

Según Manning (2002), el 90 \% de los futbolistas examinados en su estudio tienen un 2D:4D inferior a 0.98; lo cual indica que este tipo de medidas de 2D:4D permiten el desarrollo y mantenimiento de las habilidades deportivas y la eficacia del sistema cardiovascular (Manning, Stewart, Bundred \& Trivers, 2004). Además de una adecuada habilidad viso-espacial, resistencia física y velocidad, relacionando distintas variables de la condición física, como por ejemplo, equilibrio, rapidez de movimientos de piernas, flexibilidad, fuerza explosiva, fuerza estática, fuerza del tronco, fuerza funcional, entre otras, (Peeters \& Claessens,2013). Los datos de nuestro estudio coinciden con esta línea de antecedentes.

Otro análisis importante para destacar es que el 2D:4D en deportes como tenis de campo, baloncesto e incluso voleibol, mostraron un alto 2D:4D. Lo cual permite inferir que estos datos corroboran la supremacía de la técnica, lo cual es reiterado en gimnastas femeninas (Peeters \& Claessens, 2013). Lo señalado previamente por la literatura, coincide con nuestros resultados y los subconjuntos homogéneos del análisis post-hoc de Tukey-B. Así mismo, el 2D:4D se asocia con otras características como la habilidad deportiva, la masa corporal y las medidas antropométricas, entre otras características, en este tipo de deportes (Aguilera y Mesa, 2018; Cohn \& Zheng, 2011). En perspectiva de nuestros resultados, futuros trabajos deben considerar que la validez del 2D:4D como predictor de rendimiento debería limitarse a algunos deportes específicos (Moffit \& Swanik, 2011).

Excluyendo del análisis a cinco jugadores infantiles de Badminton y Tenis de mesa, al comparar las características antropométricas con deportistas de nivel mundial, se observa que, la talla fue muy inferior en los diferentes deportes evaluados (Pradas, et al., 2013) y a la posición de juego (Ramos, et al., 2018). La masa corporal es similar a la presentada por jugadores de tenis de mesa de nivel mundial como los de España, Argentina, Dinamarca y Malasia, aunque en comparación con otros deportes y posiciones se encontró un promedio muy por encima (Pradas et al., 2013; Ramos et al., 2018). El IMC se encuentra en el rango esperado ideal para este tipo de deportistas entre 23 y $30 \mathrm{~kg} / \mathrm{m}^{2}$ (Gorla, et al., 2019; Arencibia et al., 2017). La edad media de los participantes responde a la relación entre la edad y el rendimiento alcanzado (Duque y Dosil, 2017).

Si bien estos resultados aportan a la discusión del posible papel diferenciador del 2D:4D en el rendimiento deportivo y de los deportes de pelota, es importante mencionar algunas limitantes. Primero, la necesidad de un grupo control que permita esclarecer mejor las diferencias, por ejemplo, comparación con no-deportistas. Segundo, aunque tratamos de controlar la variable de un grupo de deportes (de pelota) aún se deja entrever la varianza de los deportes, reconociendo las particularidades físicas, técnicas y tácticas de cada uno. Finalmente, las medidas de auto-reporte de las variables antropométricas generan un sesgo. Dicho esto, se sugiere que sean consideradas otras oportunidades de futuros trabajos de acuerdo con estas limitaciones y la contribución a comprender la relación con el 2D:4D, así como explorar otros modelos estadísticos multivariantes que permitan representar algunas variables de control (e.g., no-deportistas o deportistas aficionados). En general, estos resultados contribuyen al proceso de detección, identificación y orientación de talentos, a partir de la evaluación de una característica estable, de fácil, rápida y económica medición.

Concluimos que hay diferencias en el 2D:4D del promedio de ambas manos. La longitud de los dedos 2Dd-i y 4Ddi muestra diferencias en los deportes de pelota, principalmente, bádminton, softbol, y squash cuyas características físico-técnicas y psicológicas son similares. Si bien no se pueden establecer puntos de corte para la detección de talentos, dadas las diferencias entre grupos étnicos encontradas por Fink et al. (2006), si queda claro que los deportistas de deportes de pelota hombres tienen un 2D:4D inferior a las mujeres y a los no deportistas.

\section{Agradecimientos y Financiación}

Los autores agradecen a los deportistas, entrenadores, federaciones y ligas deportivas. Esta investigación fue financiada por la Universidad de Caldas, identificado con el código 1159516.

\section{Contribución}

SR y AG: Concepción y Diseño del estudio. SR,AG y FA: Recolección de los datos. SR y HA-L: Análisis e interpretación de los datos. SR, AG, FA y HA-L: Escritura y Revisión del manuscrito. Todos los autores aprueban la versión final sometida.

\section{Referencias}

Aguilera. R. B.. \& Mesa. S. L. (2018). Ratio de los dedos. Posible indicador para la detección de talentos deportivos en la iniciación. OLIMPIA Revista de la Facultad de Cultura Física de la Universidad de Granma. 15(47):90-102. Disponible en: https:// dialnet.unirioja.es/servlet/articulo?codigo $=6353161$.

Arias, I., Cardoso, T. A., Aguirre-Loaiza, H., \& Arenas, J. A. (2016). Características psicológicas de rendimiento deportivo en deportes de conjunto: diferencias entremodalidad y género. Revista Psicogente, 19(35), 25-36. https://doi.org/10.17081/psico.19.35.1206

Bulatova. M.. \& Platonov. V. N. (2017). La preparación física. $4^{\mathrm{a}}$ Edición. Barcelona: Editorial Paidotribo.

Cheng. Ch. H.. Borcherng. S.. Nai. W. K.. Su. L. L.. Tsorng. H. F.. Chung. P.Ch.. Ching. Ch. Ch.. \& Mei.Ch.H. (2015). Elite Collegiate Tennis Athletes Have Lower 2D: 4D Ratios Than Those of Nonathlete Controls. Journal of Strength and Conditioning Research: 29(3) 822-825. doi.org/10.1519/ JSC.0000000000000681.

Cohen, J. (1988). Satistical Power Analysis for the Behavioral Sciences. Lawrence Erlbaum Associates. 
De Bosscher. Bingham. Shibi. van Bottenburg. de Knop. (2007). The global sporting arms race. An international comparative study on sports Policy factors leading to international sporting success. Oxford: Meyer \& Meyer Sport (UK).

Duque. I. A.. \& Dosil. J. (2017). Variables que influyen en la transición deportiva: un estudio con futbolistas en activo y exfutbolistas de la primera división de la LFP. Cuadernos de Psicología del Deporte. 17(3).13-24.

Faul, F., Erdfelder, E., Buchner, A., \& Lang, A.-G. (2009). Statistical power analyses using $G^{*}$ Power 3.1: Tests for correlation and regression analyses. Behavior Research Methods. https://doi.org/ 10.3758/BRM.41.4.1149

Ferriz. V. A.. Sellés. P. S.. García. J. M.. Cejuela.A. R. (2020). Relative age effect for talents' development in joung triathletes. Retos. 37. 27-32.

Frick. A. N.. Hull. J. M.. Manning. T. J.. \& Tomkinson. R. G (2017). Relationships between digit ratio (2D:4D) and basketball performance in Australian men. American Journal of Human Biology. 29 (3):e22937. May. doi.org/10.1002/ajhb.22937.

Fritz, C. O., Morris, P. E., \& Richler, J. J. (2012). Effect size estimates: Current use, calculations, and interpretation. Journal of Experimental Psychology: General,141(1),2-18. https://doi.org/10.1037/ a0024338

Gabbett, T. J., Sheppard, J. M., Pritchard-Peschek, K. R., Leveritt, M. D., \& Aldred, M. J. (2008). Influence of Closed Skill and Open Skill Warm-ups on the Performance of Speed, Change ofDirection Speed, Vertical Jump, and Reactive Agility in Team Sport Athletes. Journal of Strength and Conditioning Research, 22(5), 1413-1415. https:/ /doi.org/10.1519/JSC.0b013e3181739ecd

García. N. A.. \& Díaz. M. J.F. (2010). Relación entre optimismo/ pesimismo disposición al rendimiento y edad en jugadores de fútbol de competición. Revista de Iberoamericana de Psicología del Ejercicio y el Deporte. 5(1). 45-60.

Gómez. M. G. \& Sánchez. S. M. (2019). Description and differences in the psychological variables related to sports performance of triathletes and para-triathletes. Retos. 36. 22-25.

Gorla. J. I.. Nogueira. C. D.. Gonçalves. H. R.. Rosch. De F. F.. Reis. B. J.. Nunes. N.. Tafarel. P. do R. J.. Borges. M.. Brandão. V. I.. \& Labrador. R. V.(2019). Body composition and somatotype profile of football-seven brazilian players with cerebral palsy by functional classification. Retos. 35: 326-328.

Holway. F. E.. \& Guerci. G. (2012). Capacidad predictiva de los parámetros antropométricos y de maduración sobre el rendimiento en remo-ergómetro en adolescentes noveles. Apunts Medicina de l'Esport. 47(175): 99-104.

Hönekopp. J.. Bartholdt. L.. Beier. L.. \& Liebert. A. (2007). Second to fourth digit length ratio (2D:4D) an adult sex hormone levels: New data and a meta-analytic review. Psychoneuroendocrinology. 32: 313-321. doi.org/10.1016/j.psyneuen.2007.01.007.

Hsu. C. C.. Su. B.. Kan. N. W.. Lai. S. L.. Fong. T. H.. \& Chi. C. P. (2015). Elite collegiate tennis athletes have lower 2D: 4D ratios than those of nonathlete controls. Journal of Strength and Conditioning Research. 29(3). 822-825. doi.org/10.1519/ JSC. 0000000000000681.

Hull. M. J.. Schranz. N. K.. Manning. J. T.. \& Tomkinson. G. R. (2015). Relationships between digit ratio (2D:4D) and female competitive rowing performance. American Journal Humanan Biology. 27(2).157-63 doi.org/10.1002/ajhb.22627.

Kikuchi. N.. Nakazato. K.. Min. S. K.. Ueda. D.. \& Shoji. I. (2014). THE ACTN3 R577X Polymorphism is associated with muscle power in male Japanese athletes. Journal Strength Condition Research. 28(7).1783-9. doi.org/10.1519/JSC.0000000000000338.

Kim. B. T.. \& Kim. H. K. (2016). Why is digit ratio correlated to sports performance? Journal Exercise Rehabilitaion. 12(6). 515-519. doi.org/10.12965/jer.1632862.431.

Leyva de A.J.H.(2010). Selección yorientación de talentos deportivos.
Armenia: Editorial Kínesis.

Manning. J. T.. Baron. C. S.. Wheelwright. S.. \& Sanders. G. (2001). The 2nd to 4th digit ratio and autism. Developmental Medicine Child Neurology. 43(3):160-4.

Manning. J. T.. Hill. M. R. (2009). Digit ratio (2D:4D) and sprinting speed in boys. American Journal Humanan Biology. 21.210-213. doi.org/10.1002/ajhb.20855.

Manning. J. T.. Stewart.A.. Bundred. P. E.. \& Trivers. R. L. (2004). Sex and ethnic differences in 2nd to 4th digit ratio of children. Early Human Development. 80(2).161-168. doi.org/10.1016/ j.earlhumdev.2004.06.004.

Manno F.A. (2008). Measurement of the digit lengths and the anogenital distance in mice. Physiolgical Behavior. 93 (1-2):364-8. doi.org/ 10.1016/j.physbeh.2007.09.011.

McGrath, R. E. (2011). Quantitative models in psychology. In Quantitative models in psychology. American Psychological Association. https://doi.org/10.1037/12316-000

Marrodán. M.D.. Romero. J.F.. Moreno. S.. Mesa. M.S.. Cabañas. M.D. Pacheco. J.L. et al. Dinamometría en niños y jóvenes entre 6 y 18 años: valores de referencia. asociación con tamaño y composición corporal. Anales de Pediatría. 2009 70(4): 340-348. Doi: 10.1016/j.anpedi.2008.11.025.

Moffit D. M.. \& Swanik C. B. (2011). The association between athleticism. prenatal testosterone. and finger length. Journal Strength Condition Research. 25:1085-1088. doi.org/10.1519/ JSC.0b013e3181d4d409.

Paul, D. J., Gabbett, T. J., \& Nassis, G. P. (2016). Agility in Team Sports: Testing, Training and Factors Affecting Performance. In Sports Medicine (Vol. 46, Issue 3, pp. 421-442). Springer International Publishing. https://doi.org/10.1007/s40279-015-04282

Peeters. M.. \& Claessens. A. (2013). The left hand second to fourth digit ratio (2D:4D) does not discriminate world-class female gymnasts from age matched sedentary girls. PloS one. 7(6). e40270. http://dx.doi.org/10.1371/journal.pone.0040270.

Pradas. de la F. F.. González. J. J. A.. Molina. S. E.. \& Castellar. O. C. (2013). Características Antropométricas. Composición Corporal y Somatótipo de Jugadores de Tenis de Mesa de Alto Nivel. International Journal Morphology. 31(4): 1355-1364. doi.org/ 10.4067/S0717-95022013000400033.

Ramos. S. F.. Camina. M. M.A..Alonso. de la T. S. R.. Redondo del R. P.. \& de Mateo. S. B. (2018). Composición corporal y somatótipo por posición de juego en balonmano profesional masculino. Revista Internacional de Medicina y Ciencias de la Actividad Física yel Deporte. 18(69): 91-102. doi.org/10.15366/rimcafd2018.69.006.

Ruiz. B. R.. \& García. N. A. (2013). Personalidad. edad y rendimiento deportivo en jugadores de fútbol desde el modelo de Costay McCrae. Analales de Psicología. 29(3): 642-655 doi.org/10.6018/ analesps.29.3.175771.

Sudhakar. H.H.. Majumdar. P.. Umesh. V.. \& Panda. K. (2014). Second to fourth digit ratio is a predictor of sporting ability in elite indian male kabaddi players. Asian Journal Sports Medicine. 5(3):e23073. doi.org/10.5812/asjsm.23073.

Tamiya R.. Lee S.Y.. Ohtake F. (2016) Second-to-Fourth Digit Ratio and the Sporting Success of Sumo Wrestlers. In: Ikeda S.. Kato H.. Ohtake F.. Tsutsui Y. (eds) Behavioral Economics of Preferences. Choices. and Happiness. DOI https://doiorg.bdigital.ces.edu.co:2443/10.1007/978-4-431-55402-8_23

Valdés. B.P.. Salvador. S. N.. Godoy. C.A.. Carmona.L. M. I.. Fernández. J. J.. \& Durán. A. S. (2015). Somatotipo. estado nutricional y nivel de glucemia de estudiantes de educación física. Nutrición Hospitalaria. 32(3): 1261-1266. doi.org/10.3305/nh.2015.32.3.9402.

Volkov. V. M.. Filin. V.P. (1988). Selección deportiva. Moscú: Editorial Pueblo y Educación.

Weineck. J.(2005). Entrenamiento Total. Barcelona: Editorial Paidotribo. 